\title{
ERYTHRO-9-(2-HYDROXY-3-NONYL)ADENINE (EHNA) BLOCKS DIFFERENTIATION AND MAINTAINS THE EXPRESSION OF PLURIPOTENCY MARKERS IN HUMAN EMBRYONIC STEM CELLS
}

\author{
Peter Burton ${ }^{1}$, David R. Adams ${ }^{2}$, Achamma Abraham ${ }^{2}$, Robert W. Allcock ${ }^{2}$, Zhong Jiang ${ }^{2}$, \\ Angela McCahill ${ }^{1}$, Jane Gilmour ${ }^{1}$, John McAbney ${ }^{1}$, Alexandra Kaupisch ${ }^{1}$, Nicole M. Kane ${ }^{3}$, \\ George S. Baillie ${ }^{1}$, Andrew H. Baker ${ }^{3}$, Graeme Milligan ${ }^{1}$, Miles D. Houslay ${ }^{1}$ and Joanne C. \\ Mountford ${ }^{1}$.
}

From ${ }^{1}$ Neuroscience and Molecular Pharmacology, Faculty of Biomedical and Life Sciences (FBLS), and ${ }^{3}$ Glasgow Cardiovascular Research Centre, Faculty of Medicine, University of Glasgow, Glasgow G12 8QQ, Scotland, UK; ${ }^{2}$ Department of Chemistry, Heriot-Watt University, Riccarton Campus, Edinburgh EH14 4AS, Scotland, UK

Short title: EHNA maintains hESC pluripotency

Address correspondence to Dr J C Mountford, Neuroscience and Molecular Pharmacology, Faculty of Biomedical and Life Sciences (FBLS), University of Glasgow, Glasgow G12 8QQ Scotland, UK. Email : j.mountford@bio.gla.ac.uk; Phone :+44 1413307212

\begin{abstract}
Abbreviations: hESCs, Human embryonic stem cells; EHNA, erythro-9-(2-hydroxy-3-nonyl)adenine; bFGF, basic fibroblast growth factor; ADA, Adenosine deaminase; LIF, leukaemia inhibitory factor; GSK $3 \beta$, glycogen synthase kinase 3 beta; PDE, phosphodiesterases; IBMX, isobutylmethylxanthine; AICAR, 5-aminoimidazole-4-carboxamide riboside; ABT-702, 4-amino-5-(3-bromophenyl)-7-(6morpholinopyridin-3-yl)pyrido[2,3-d] pyrimidine; NECA, 5'- $N$-ethylcarboxamidoadenosine, THEO, theophylline; NF, exogenous FGF-free medium; NFE, exogenous FGF-free medium containing 10uM EHNA.
\end{abstract}

Keywords: Human embryonic stem cells; EHNA; Differentiation; ell culture; Adenosine Deaminase; Fibroblast Growth Factor 


\section{SYNOPSIS}

Human embryonic stem cells (hESCs) have enormous potential for use in pharmaceutical development and therapeutics, however to realise this potential there is a requirement for simple and reproducible cell culture methods that provide adequate numbers of cells of suitable quality. We have discovered a novel way of blocking the spontaneous differentiation of hESCs in the absence of exogenous cytokines by supplementing feeder-free conditions with erythro-9-(2hydroxy-3-nonyl)adenine (EHNA), an established inhibitor of adenosine deaminase (ADA) and cyclic nucleotide phosphodiesterase- 2 (PDE2). hESCs maintained in feeder-free conditions with EHNA for more than 10 passages showed no reduction in hESC associated markers including NANOG, POU5F1 and SSEA 4 compared to cells maintained in feeder-free conditions containing basic fibroblast growth factor (bFGF). Spontaneous differentiation was reversibly suppressed by the addition of EHNA, but upon removing EHNA hESC populations underwent efficient spontaneous, multi-lineage and directed differentiation. EHNA also acts as a strong blocker of directed neuronal differentiation. Chemically distinct inhibitors of ADA and PDE2 lacked the capacity of EHNA to suppress hESC differentiation, suggesting that the effect is not driven by inhibition of either ADA or PDE2. Preliminary structure activity relationship analysis found the differentiation blocking properties of EHNA to reside in a pharmacophore comprised of a close adenine mimetic with an extended hydrophobic substituent in the 8- or 9-position. We conclude that EHNA and simple 9-alkyladenines can block directed neuronal and spontaneous differentiation in the absence of exogenous cytokine addition, and may provide a useful replacement for bFGF in large scale or cGMP compliant processes.

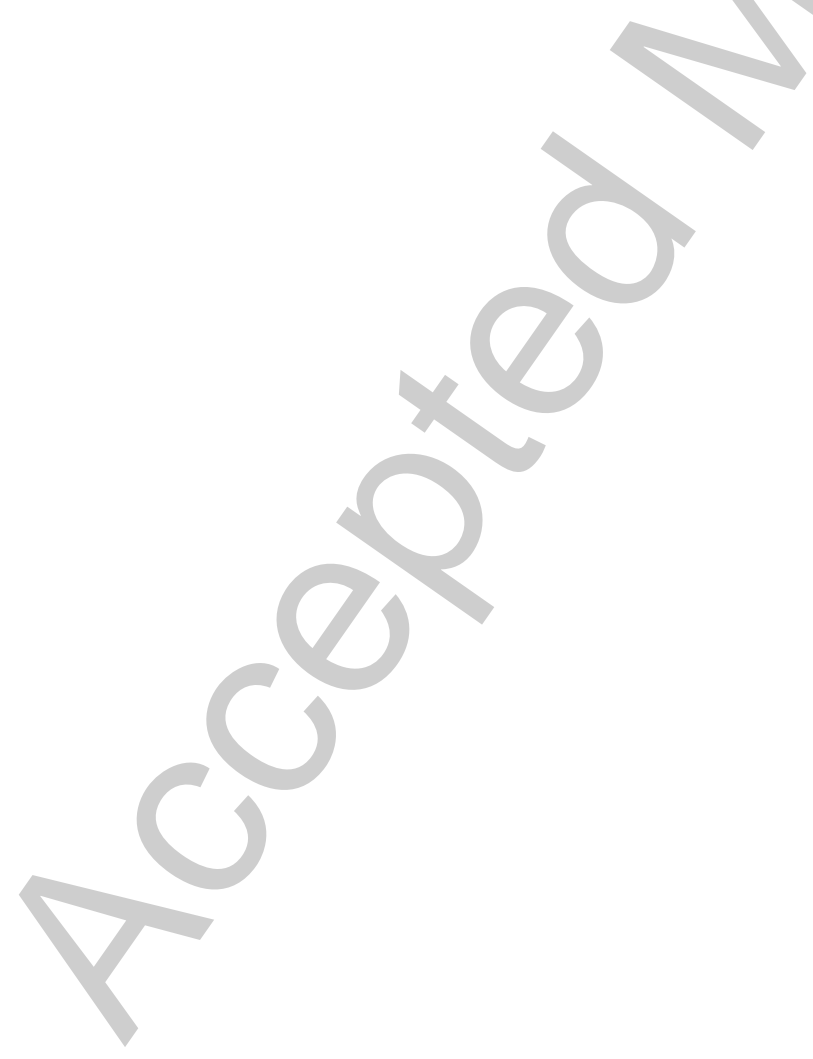


Burton et al.

\section{INTRODUCTION}

Human embryonic stem cells (hESCs) can be maintained in an undifferentiated state whilst remaining capable of differentiating to all cell types in the body [1,2], as such, they have huge potential both as research tools and for cell therapies. In order to fully exploit this potential we must understand how to efficiently maintain hESCs in this self-renewing, pluripotent state. A large body of evidence indicates that the three transcription factors POU5F1, NANOG and SOX2 constitute the core transcriptional regulatory circuit that determines the fate of ESCs [3-7]. Expression levels of POU5F1/NANOG/SOX2 and their target genes are crucial in imposing stem cell identity and controlling differentiation [8], and therefore it is critical for any culture system to maintain appropriate expression of this network. Methods to suppress spontaneous differentiation and maintain pluripotent hESCs vary greatly and utilise either feeder cells, feeder-conditioned media [9] or different combinations of cytokines (including high concentrations of bFGF $[10,11]$, TGF$\beta /$ NODAL/ACTIVIN signalling molecules [12-14] or bone morphogenic protein (BMP) suppressors such as noggin $[10,15])$.

Small-molecule chemicals have been used in attempts to replace exogenous cytokines, in particular glycogen synthase kinase 3 beta (GSK3 $\beta$ ) inhibitors, which stimulate the canonical Wnt/ $\beta$-catenin axis, have been widely used to maintain pluripotency in mESC and in some hESC studies [16]. Additionally, Ying et al found that stimulation of cells by exogenous growth factors was not required for the maintenance of pluripotency in mouse ESCs, instead suppression of pro-differentiation signals - by inhibiting mitogen-activated protein kinase (MAPK), GSK-3 $\beta$ and FGF signaling - was sufficient to maintain self-renewal and pluripotency [17]. Thus, rather than using combinations of complex growth factors to stimulate self-renewal, protecting the cells from pro-differentiation factors or using small-molecule inhibitors of differentiation may provide viable alternatives for the maintenance of hESCs. Given the importance of the core transcriptional network in both maintaining and inducing pluripotency, the discovery of small molecules that can maintain the expression of these transcription factors, either in the presence of differentiation cues or in the absence of maintenance factors such as exogenous FGF, would be a valuable asset to the field and a further step towards unraveling the mechanisms of pluripotency.

Cyclic nucleotides are pivotal second messengers that influence a number of critical events including cell cycle and differentiation processes. Cyclic nucleotide phosphodiesterases (PDEs) provide the sole means of degrading cAMP and cGMP in cells with selective inhibitors shown to provide powerful reagents to manipulate specific cellular events and act as therapeutic agents [18]. With this in mind we analysed the effect of a range of inhibitors selective for major PDE families on hESC differentiation. From this we discovered that erythro-9-(2-hydroxy-3-nonyl)adenine (EHNA), an inhibitor of both the dual specificity cAMP/cGMP-phosphodiesterase, PDE2 [19] and adenosine deaminase (ADA) [20], maintains the expression of hESC pluripotency markers even when cells are cultured under differentiating conditions. Moreover, EHNA also prevents differentiation and maintains pluripotency when exogenous FGF is removed from a supportive culture system. It thus provides a simple chemical entity that is capable of blocking the differentiation of hESCs.

\section{EXPERIMENTAL}

\section{Cell culture and differentiation}

The SA121 hES cell line [21], (Cellartis AB, Dundee, UK) was cultured in feeder-free conditions on fibronectin and enzymatically passaged with TrypLE Select (Invitrogen). The fully supportive media used to maintain the hESCs was a 1:1 mixture of defined media [22] and conditioned VitrohES (VitroLife, Gothenburg, Sweden). VitrohES was conditioned for $24 \mathrm{~h}$ on mitotically inactivated mouse embryonic fibroblasts (MEF) with no addition of FGF. SA461 hES cells [21] (Cellartis AB) were maintained on MEFs and used to test whether EHNA supports the change from feeders to feeder-free 
culture (Figure 1a). Routine maintenance used bFGF (Invitrogen) at a final concentration of $10 \mathrm{ng} / \mathrm{ml}$ unless stated otherwise. For passive differentiation cells were passaged onto Matrigel (BD Biosciences, Oxford, UK) in fully supportive media, changed to defined medium supplemented with experimental components $24 \mathrm{~h}$ later and thence changed daily. Cytogenetic analyses confirmed a normal karyotype in cells grown in the presence of EHNA for either 10 or 20 passages, or with HWC57 or HWC64 for 22 passages (Supplemental Figure 7).

Erythro-9-(2-hydroxy-3-nonyl)adenine (EHNA) was used at $10 \mu \mathrm{M}$ unless otherwise stated (Merck Chemicals Ltd., Nottingham, UK). Other compounds were purchased from Merck or Sigma-Aldrich except BAY-60-7550 (Axxora). A range of EHNA analogues were synthesized; compound structures and synthetic procedures are detailed in the Supplemental Chemistry section. Neuronal differentiation was carried out as described previously [23]. Briefly, cells were seeded onto Matrigel (BD Biosciences) in 1:1 mix of Advanced D-MEM/F12 containing N2 supplement, and Neurobasal supplemented with B27 (all Invitrogen) containing $100 \mathrm{ng} / \mathrm{ml}$ mouse recombinant Noggin (R\&D Systems). Cells were allowed to reach confluence and passaged using collagenase (Invitrogen) after 2 weeks.

\section{Quantitative PCR}

RNA was extracted and cDNA prepared as previously described [24]. Gene-specific assays were used for DNMT3b, KLF4, TEAD4 and POU5F1 (Applied Biosystems (AB)); other primer and probe sets were from MWG (Ebersberg, Germany). Biological and technical triplicates were performed for each sample, standardised to GAPDH and relative expression values calculated. The human stem cell pluripotency Taqman Low Density Array (TLDA) was also used with the 7900HT system (AB).

\section{Immunocytochemistry}

Cells were incubated at $4{ }^{\circ} \mathrm{C}$ overnight with the following antibodies in $10 \%$ goat serum (SigmaAldrich)/PBS: mouse anti-POU5F1 (1:250) (Santa Cruz Biotech., California, USA), rabbit anti-PAX6 (1:1000) (Millipore Ltd, Herts, UK), mouse anti $\beta$-tubulin III (1:1000), mouse anti-Alpha-feta-protein (AFP) (1:400) (Sigma-Aldrich), mouse anti-muscle-specific actin (SMA) (1:50) (Dako, Denmark), SSEA1 (1:5), SSEA3 (1:5) (Hybridoma Bank University of Iowa), SSEA4 (1:5) (Hybridoma Bank University of Iowa), Tra1-60 (1:200) and Tra1-80 (1:200) (Santa Cruz Biotech.). Secondary antibodies were Alexa fluor 555 goat anti-rabbit IgG (1:400) or Alexa fluor 488 goat anti-mouse $\operatorname{IgG}(1: 400)$ and cells were mounted using Prolong Gold containing DAPI (all Invitrogen). All immunofluorescence was visualised and captured using Zeiss Axiovision image analysis system.

\section{RESULTS}

EHNA prevents differentiation and maintains the expression of stem cell markers in the absence of exogenous FGF during feeder-free growth.

The capacity of EHNA to maintain stem cell characteristics and block spontaneous differentiation in feeder-free and FGF-free conditions was evaluated by incubating hESCs (Cellartis line SA121) [21] in standard feeder-free conditions without exogenous FGF (including conditioned medium made without the addition of exogenous FGF) but supplemented with $10 \mu \mathrm{M}$ EHNA (No FGF/+EHNA medium; NFE). Cells were initially seeded from a trypsin passage of a standard, FGF-containing, feeder-free culture. Control cells grown without FGF or EHNA (No FGF medium; NF) had reduced stem cell marker (NANOG and POU5F1) expression by passage 3 and showed clear differentiated morphology by passage 8-10, whereas those grown in the presence of EHNA retained a normal stem cell morphology and marker expression to at least passage 10 (Figure 1a) and, in extended experiments, for as long as 30 passages. In addition to expressing POU5F1, cells maintained in EHNA for 10 passages also expressed the cell surface markers SSEA3, SSEA4, TRA-160 and TRA-180, but were negative for the differentiation marker SSEA1 (Figure 1b). This marker profile strongly indicated the maintenance of a pluripotent phenotype. 
To determine if prior adaptation to feeder-free conditions is required for EHNA to block the differentiation of hESCs in the absence of FGF, a culture of the hESC line SA461 was transferred directly from a supportive MEF feeder layer, using manual dissection, to NFE medium. After multiple passages the EHNA-containing cultures were still almost $100 \%$ positive for POU5F1 whereas POU5F1 staining in the cultures grown in NF medium was minimal (Figure 1a). This indicates that pre-adaptation to feeder-free conditions was not required for EHNA to block the differentiation of hESCs in the absence of FGF and that the effect of EHNA can be seen in a second hESC line..

Real time qRT-PCR analysis showed that at passage 8 in NF medium cells had greatly down-regulated the expression of NANOG, POU5F1 and SOX2 in comparison to hESCs grown in standard feederfree culture (containing FGF), or to cells grown with EHNA (NFE) (Figure 1c). The NF-cultured cells also showed an increase in the expression of a variety of differentiation markers from multiple germ layers, particularly those associated with trophoectoderm formation (Supplemental Figure 1). In contrast, EHNA (NFE medium) maintained an expression profile similar to that of cells in standard feeder-free culture and maintained that pattern for at least 30 passages (Supplemental Figure 2).

\section{Cells maintained in EHNA retain the capacity for in vitro differentiation to all three germ layers.}

It is important that any component of stem cell medium that maintains a pluripotent phenotype does not permanently block differentiation of the cells. Therefore, to determine if chronic exposure to EHNA still allowed for subsequent differentiation, cells that had been in the EHNA-containing, NFE medium for either 10 passages (Figure 2) or $>20$ passages (Supplemental Figure 3) were allowed to differentiate passively. After 4 weeks cells were analysed for PAX6 (ectoderm), alpha-feta protein (AFP) (endoderm) and smooth muscle actin (SMA) (mesoderm) in order to detect differentiation to all three germ layers. Cells staining positive for three germ layers were readily detected (Figure 2 and Supplemental Figure 3). This indicates that, at least in vitro, cells grown in EHNA for over 10 passages retain functional pluripotency.

In order to understand whether pre-treatment with EHNA has any effect on the dynamics of induced, rather than spontaneous, differentiation cells that had been maintained in either EHNA or FGF for five passages were transferred into neuronal differentiation conditions [23]. Analysis over the time-course demonstrated that expression of PAX6 increased and NANOG decreased similarly in both EHNAtreated and control cultures, although we did observe that there was slight retardation of the NANOG down-regulation in EHNA-maintained cells (Figure 3a-b). Immunofluorescent staining at the 16 day time point also indicated that cells grown previously on EHNA express PAX6 protein and downregulate POU5F1 (Figure 3c-d).

\section{EHNA maintains the expression of the core stem cell transcriptional factors in differentiating conditions.}

In order to test the direct effect of EHNA in maintaining pluripotent genes and blocking differentiation, cells maintained in full feeder-free media were subjected to neuronal differentiation conditions in the presence of various doses of EHNA throughout the neuronal differentiation. As illustrated in Figure 4a, EHNA caused a dose-dependent reduction in the proportion of cells staining positively for PAX6 with $10 \mu \mathrm{M}$ EHNA completely eliminating PAX6. Additionally, qRT-PCR analysis (Figure 4b-e), showed that the pluripotent markers NANOG, POU5F1 and ZFP42 were all maintained by the presence of EHNA. This ability of EHNA to block differentiation was sustained over at least 6 weeks of neuronal induction (Figure 4f). Thus, EHNA exerts a dramatic antidifferentiation effect even in the presence of established differentiating conditions.

\section{EHNA does not suppress hESC differentiation by inhibition of PDE2.}

In order to investigate the basis for EHNA's anti-differentiation effect, we set out to exploit the capacity of EHNA to suppress passive differentiation in defined media. We evaluated the maintenance of NANOG and suppression of PAX6 expression as means of defining whether compounds with related enzyme-inhibitory activity to EHNA acted similarly on hESCs. In this regard, EHNA is an established inhibitor of both adenosine deaminase [20] and PDE2 [19, 25]. In order to evaluate whether the effect of EHNA was due to PDE2 inhibition, the specific PDE2 inhibitor, BAY-60-7550 
(BAY) $[26,27]$ and the pan-cyclic nucleotide PDE inhibitor, 3-isobutyl-1-methylxanthine (IBMX) [25], were each separately added to cultures undergoing passive differentiation (as above). In contrast to EHNA, treatment with neither BAY nor IBMX maintained stem cell marker expression in differentiating conditions (Figure 5a); nor did they inhibit PAX6 induction (Figure 5b). This strongly suggests that PDE2 inhibition is not the mechanism whereby EHNA maintains hESC pluripotency.

\section{EHNA does not suppress hESC differentiation by inhibition of adenosine deaminase.}

EHNA is also an established ADA inhibitor and, as such, it causes the accumulation of adenosine and deoxyadenosine, resulting in pleiotropic effects upon the cell [20, 28-32]. To determine whether the effects of EHNA are mediated by phosphorylated metabolites of adenosine, an adenosine kinase inhibitor, ABT-702 (AKI), was used in conjunction with EHNA. After 2 weeks under passive differentiation conditions the effects of EHNA were not reduced by the concurrent inhibition of adenosine kinase (Figure 5c). Additionally, stimulation of AMPK with 5-aminoimidazole-4carboxamide riboside (AICAR) did not mimic the effect of EHNA. Thus, we concluded that phosphorylation of adenosine is not necessary for the maintenance of pluripotency markers by EHNA.

Accumulation and export of adenosine and deoxyadenosine can, in principle, lead to activation of the adenosine receptors. To investigate whether adenosine receptor activation is responsible for the maintenance of hESC pluripotency markers by EHNA, we evaluated whether $5^{\prime}-\mathrm{N}$ ethylcarboxamidoadenosine (NECA), a pan-adenosine receptor agonist, and the non-selective PDE inhibitor and adenosine receptor antagonist, theophylline, could ablate the effect of EHNA. Neither agent, had any effect on the expression of hESC pluripotency markers in the presence or absence of EHNA (Figure 5c). Similarly, targeting individual adenosine receptor subtypes with specific antagonists in addition to EHNA had no effect (Figure 5d). Hence, adenosine receptor activity is unlikely to underpin EHNA's action on hESCs.

To further investigate whether the effect of EHNA might still be driven by ADA inhibition, we sourced or synthesised a panel of structurally diverse compounds (Figure 6; Supplemental Figures 4 and 5) known to possess widely varying degrees of ADA-inhibitory activity. Critically, there was no correlation between ADA-inhibitory activity and the capacity of these compounds to maintain pluripotency-associated factors and to suppress PAX6 expression. Some compounds with potent ADA-inhibitory activity failed to show any EHNA-like effect. Pentostatin, an exceptionally potent ADA-inhibitor $\left(K_{\mathrm{i}}=2.5 \mathrm{pM}\right.$ for ADA) [33], lacked any effect at concentrations of up to $100 \mu \mathrm{M}$ even in combination with the PDE2 inhibitor, BAY-60-7550 (data not shown). Similarly, erythro-1-(2hydroxynonan-3-yl)-1H-imidazole-4-carboxamide (HWC-36), an analogue of EHNA with a truncated adenine ring, showed no capacity to maintain NANOG or to suppress PAX6 expression at a concentration of $10 \mu \mathrm{M}$, despite possessing potent ADA-inhibitory action $\left(K_{\mathrm{i}}=35 \mathrm{nM} v s \mathrm{nM}\right.$ for EHNA) [34]. However, a series of 2-alkyl-2H-pyrazolo[3,4- $d$ ]pyrimidin-4-amine ADA inhibitors described by Da Settimo et al. [35] did exhibit EHNA-like maintenance activity. The octyl, nonyl, decyl and undecyl analogues in this series (Figure 6 and Supplemental Figure 4; respectively HWC41, HWC-31, HWC-06 and HWC-33), with reported ADA-inhibitory $K_{\mathrm{i}}$ values of 530, 8.1, 0.13 and $0.47 \mathrm{nM}$, exhibited pronounced maintenance of the pluripotency marker, NANOG, coupled with a suppression of the differentiation marker, PAX6, when tested at $10 \mu \mathrm{M}$ concentrations. Da Settimo et al [35] found the 1-alkyl-substituted isomers of their 2-alkylpyrazolopyrimidine series lacked significant ADA-inhibitory activity. However, we found that the octyl- and nonyl- analogues in the non-ADA-inhibitory 1-alkylpyrazolopyrimidine series (respectively HWC-40 and HWC-30) retained the differentiation-suppressive properties of their 2-alkyl isomers and of EHNA. As shown in Figure 7, HWC-30, like HWC-31 and HWC-33, maintained the expression of pluripotency genes whilst suppressing PAX6. Collectively these data strongly suggest that the action of EHNA on hESCs is not driven by ADA inhibition.

To investigate the possibility that EHNA and other active compounds may act through a protein kinase-inhibitory mode, EHNA, HWC-5 and HWC-6 were evaluated using the Protein Kinase Panel service of the MRC National Centre for Protein Kinase Screening (University of Dundee, UK). When tested at $30 \mu \mathrm{M}$, none of these compounds significantly inhibited any of the 80 kinases of the panel 
(Supplemental Figure 6). This included GSK-3ß[16], a number of ERK/MAPK pathway components [36] and FGF-R1 [37], which have all previously been shown to be important in hESC pluripotency.

\section{Structure activity relationships for the differentiation blocking activity of EHNA}

As EHNA did not appear to exert its action on hESCs through inhibition of either PDE2 or ADA, we undertook a preliminary structure activity relationship analysis by evaluating a wider set of EHNA analogues. The compounds assessed, Figure 6 (and detailed in full in the Supplemental Material) show that maintenance of NANOG and suppression of PAX6 requires a compound structure comprising a close adenine mimetic with an extended hydrophobic substituent in the 8- or 9-position. We found that the EHNA structure could be simplified by removal of the hydroxy group from the nonanol side chain, and deoxy-EHNA (HWC-57) retains activity comparable to EHNA itself. As a further step, we replaced the branched hydrocarbon chain of the latter compound with a straight chain $n$-decyl group to provide a compound [9-(decan-1-yl)adenine, HWC-64] that also retained the full action of EHNA on hESCs. In fact cells grown for 10 passages in FGF-free feeder-free conditions, but supplemented with either HWC-57 $(10 \mu \mathrm{M})$ or HWC-64 $(10 \mu \mathrm{M})$ show an equivalent morphology (Figure 8a) and gene expression pattern (Figure $8 \mathrm{~b}$ ) to those grown in FGF-containing supportive media. Cells without either FGF or HWC-57/HWC-64 show a strong differentiated morphology by passage 4 (Figure 8a). These data indicate that HWC-57 and HWC-64 are functionally equivalent to EHNA with regard to maintaining the markers of pluripotency in the absence of FGF.

The recently disclosed EHNA-ADA co-crystal structure reveals that its hydroxyl side chain contributes an important hydrogen bond to a histidine residue within the catalytic pocket [38]. The redundancy of this hydroxyl group in regard to the hESC differentiation blocking properties further supports the contention that this activity is not mediated through ADA inhibition. Thus, neither of the two established activities of EHNA, namely ADA inhibition and PDE2 inhibition, appear to be responsible for the maintenance of hESC pluripotency and suppression of differentiation. This implies a novel mechanism of action for which, although currently unidentified, we have provided preliminary structure activity relationships (SAR) by studying a range of EHNA analogues.

\section{DISCUSSION}

The regulation and maintenance of pluripotency in hESCs is still far from fully understood and there are multiple methods for cultivating hESCs in a pluripotent state. Traditionally hESCs have been cultivated on a mouse embryonic feeder layer [2] with further recent advances resulting in feeder-free culture systems on extracellular matrix proteins, which may be recombinant or extracted $[10-12,14$, 15]. However, hESCs cultured in these feeder-free systems lack the mainly undefined stimuli from the feeder cells that maintain these cells in a self-renewing, pluripotent state and therefore, additional recombinant growth factors must be added to replace the contribution of the feeder cells. The only growth factor that has been shown to be essential for the maintenance of pluripotency in the absence of feeder-layers or conditioned media is basic FGF (bFGF, FGF-2) [10, 37]. However other factors, including TGF $\beta$, are also commonly used to either remove or reduce the need for high concentrations of bFGF and/or conditioned media [12-14]. Such recombinant growth factors have a plethora of effects and, when considering the potential for using pluripotent cell-derived tissues in the clinic, are difficult to obtain from current good manufacturing practice-qualified sources. Simple small molecule alternatives to growth factors for maintaining pluripotent hESCs would not only be more amenable to current good manufacturing practice-compliant use, but are likely to provide powerful new tools for trying to uncover the molecular basis of pluripotency.

We have demonstrated here that the spontaneous differentiation of hESCs in the absence of either feeder cells or exogenous cytokines can be blocked by the addition of EHNA. We have also shown that EHNA is sufficiently supportive in that cells could be directly switched from feeders into feederfree culture without pre-adaptation to feeder-free culture containing exogenous FGF. Importantly, we found that chronic EHNA treatment did not 'lock' hESCs in an undifferentiated state, as the EHNAtreated cells were capable of multi-lineage differentiation when EHNA was removed. However, suppression of differentiation was sufficiently robust that when cells were switched into neuronal 
inducing conditions, the continued addition of EHNA blocked PAX6 expression and maintained the pluripotent morphology and the markers NANOG, POU5F1 and ZPF42. Furthermore, through our structure activity relationship analysis, we discovered that simple 9-alkyladenines, such as HWC-57 and HWC-64, possess activity comparable to EHNA. These robust, stable compounds are readily prepared from commercially available starting materials at low cost in just two synthetic steps, and, as such, offer a very attractive alternative to the use of bFGF in large scale applications.

EHNA has a number of reported effects including the inhibition of PDE2 [19], but as we were unable mimic the effect of EHNA with either a structurally dissimilar PDE2 specific inhibitor (BAY-607550) [26] or the non-specific cyclic nucleotide PDE inhibitor IBMX [25] we can conclude that PDE2 inhibition does not account for the effect of EHNA in hESCs. EHNA has also been reported to reduce intracellular trafficking by inhibition of dynein. However, concentrations in the order of $100 \mu \mathrm{M}$ are typically used for inhibiting dynein, because the $\mathrm{EC}_{50}$ value for this lies between $0.23 \mathrm{mM}$ and $\sim 1 \mathrm{mM}$ $[39,40]$. As we used EHNA at a maximum concentration of $10 \mu \mathrm{M}$ and see significant effects at 1 $\mu \mathrm{M}$, we believe that it is unlikely that EHNA acts through effects on dynein in hESCs. The inhibition of ADA by EHNA was a particularly interesting possibility because a deficiency of ADA can cause a multitude of effects on the cell including apoptosis, the inhibition of transmethylation and an accumulation of adenosine that can subsequently lead to the activation of AMPK and adenosine receptors $[30,41]$. We therefore investigated whether the ADA-inhibitory activity of EHNA was essential to its capacity to block differentiation in hESCs via the accumulation of adenosine and autocrine activation of cell-surface receptors, but we could not mimic the effect of EHNA using adenosine receptor agonists. Neither could we ablate the effect of EHNA with adenosine receptor antagonists. A build up of the phosphorylated metabolites of adenosine was also not responsible for the EHNA effect. To further investigate the possibility of ADA inhibition being responsible we used a panel of established adenosine deaminase inhibitors that are chemically distinct from EHNA, and clearly demonstrated (Figures $6 \& 7$ ) that there is no link between the two activities. This leads us to believe that EHNA has an unidentified biological activity that is responsible for its ability to prevent hESC differentiation.

It is an interesting proposition that EHNA may be able to maintain pluripotent marker expression by blocking differentiation. Ying et al. proposed that mESCs actually exist in a basal self-maintaining state that can be perpetuated if shielded from differentiation-inducing signals [17]. Unlike mESCs, many of the intracellular signalling pathways in hESCs are as yet unidentified. Nevertheless, it is probable that they are conceptually similar to mESCs in that pluripotency can be maintained if the differentiating signals are removed or blocked. Our data show that EHNA is an efficient blocker of induced neuronal differentiation and of other lineages generated as cells differentiate passively after the removal of bFGF. It should also be noted that although feeder-free, the culture system contains a conditioned medium component. Therefore, whilst EHNA is an effective replacement for exogenous bFGF there may be a combinatorial effect with other conditioning factors in the medium.

It is therefore possible that EHNA might be used to maintain hESCs in a self-renewing ground state, regulated by NANOG expression, as has been described for mESCs treated with inhibitors of both GSK-3 $\beta$ and MAPK $[17,42]$. This hypothesis is supported by our finding that cells maintained in EHNA retained the expression of the core transcription factors NANOG, POU5F1 and SOX 2 to the same degree as those maintained by bFGF, whereas in control cultures from which bFGF was omitted these factors were dramatically down-regulated. Initial reports suggested that GSK3 $\beta$ inhibitors may have the same capacity to maintain self-renewal in hESCs as in mESCs [16]. In order to exclude the possibility that EHNA was acting by directly inhibiting common protein kinase intracellular signalling pathways, we subjected the molecule to a protein kinase screen and found that it did not significantly inhibit any of the kinases evaluated, including GSK-3 $\beta$, a number of ERK/MAPK pathway components and the FGF receptor, FGF-R1.

In summary, we have found that a small-molecule additive, EHNA, blocks the differentiation of hESCs in the absence of exogenous cytokines or feeder cells and can be used during routine enzymatic passage of the cells without the loss of multi-lineage differentiation potential. We have defined a 
pharmacophore for this action of EHNA, which is independent of its established PDE2- and ADAinhibitory activities, and identified simpler compounds that possess comparable activity. The capacity of EHNA and these derivates to block hESC differentiation may make them useful tools for the manipulation of $\mathrm{hESCs}$ and provide an important set of reagents for characterising pathways involved in the maintenance of pluripotency or suppression of differentiation in these cells. 


\section{References.}

1 Reubinoff, B. E., Pera, M. F., Fong, C. Y., Trounson, A. and Bongso, A. (2000) Embryonic stem cell lines from human blastocysts: somatic differentiation in vitro. Nat Biotechnol. 18, 399-404

2 Thomson, J. A., Itskovitz-Eldor, J., Shapiro, S. S., Waknitz, M. A., Swiergiel, J. J., Marshall, V. S. and Jones, J. M. (1998) Embryonic stem cell lines derived from human blastocysts. Science. 282, 1145-1147

3 Avilion, A. A., Nicolis, S. K., Pevny, L. H., Perez, L., Vivian, N. and Lovell-Badge, R. (2003) Multipotent cell lineages in early mouse development depend on SOX2 function. Genes Dev. 17, 126-140

4 Boyer, L. A., Lee, T. I., Cole, M. F., Johnstone, S. E., Levine, S. S., Zucker, J. P., Guenther, M. G., Kumar, R. M., Murray, H. L., Jenner, R. G., Gifford, D. K., Melton, D. A., Jaenisch, R. and Young, R. A. (2005) Core transcriptional regulatory circuitry in human embryonic stem cells. Cell. 122, 947-956

5 Chickarmane, V., Troein, C., Nuber, U. A., Sauro, H. M. and Peterson, C. (2006) Transcriptional dynamics of the embryonic stem cell switch. PLoS Comput Biol. 2, e123

6 Hart, A. H., Hartley, L., Ibrahim, M. and Robb, L. (2004) Identification, cloning and expression analysis of the pluripotency promoting Nanog genes in mouse and human. Dev Dyn. 230, 187-198

7 Mitsui, K., Tokuzawa, Y., Itoh, H., Segawa, K., Murakami, M., Takahashi, K., Maruyama, M., Maeda, M. and Yamanaka, S. (2003) The homeoprotein Nanog is required for maintenance of pluripotency in mouse epiblast and ES cells. Cell. 113, 631-642

8 Niwa, H., Miyazaki, J. and Smith, A. G. (2000) Quantitative expression of Oct-3/4 defines differentiation, dedifferentiation or self-renewal of ES cells. Nat Genet. 24, 372-376 9 Xu, C., Inokuma, M. S., Denham, J., Golds, K., Kundu, P., Gold, J. D. and Carpenter, M. K. (2001) Feeder-free growth of undifferentiated human embryonic stem cells. Nat Biotechnol. 19, 971-974

10 Xu, R. H., Peck, R. M., Li, D. S., Feng, X., Ludwig, T. and Thomson, J. A. (2005)

Basic FGF and suppression of BMP signaling sustain undifferentiated proliferation of human ES cells. Nat Methods. 2, 185-190

11 Yao, S., Chen, S., Clark, J., Hao, E., Beattie, G. M., Hayek, A. and Ding, S. (2006) Long-term self-renewal and directed differentiation of human embryonic stem cells in chemically defined conditions. Proc Natl Acad Sci U S A. 103, 6907-6912

12 Beattie, G. M., Lopez, A. D., Bucay, N., Hinton, A., Firpo, M. T., King, C. C. and Hayek, A. (2005) Activin A maintains pluripotency of human embryonic stem cells in the absence of feeder layers. Stem Cells. 23, 489-495

13 James, D., Levine, A. J., Besser, D. and Hemmati-Brivanlou, A. (2005)

TGFbeta/activin/nodal signaling is necessary for the maintenance of pluripotency in human embryonic stem cells. Development. 132, 1273-1282

14 Vallier, L., Alexander, M. and Pedersen, R. A. (2005) Activin/Nodal and FGF pathways cooperate to maintain pluripotency of human embryonic stem cells. J Cell Sci. 118, 4495-4509

15 Wang, G., Zhang, H., Zhao, Y., Li, J., Cai, J., Wang, P., Meng, S., Feng, J., Miao, C., Ding, M., Li, D. and Deng, H. (2005) Noggin and bFGF cooperate to maintain the pluripotency of human embryonic stem cells in the absence of feeder layers. Biochem Biophys Res Commun. 330, 934-942

16 Sato, N., Meijer, L., Skaltsounis, L., Greengard, P. and Brivanlou, A. H. (2004) Maintenance of pluripotency in human and mouse embryonic stem cells through activation of Wnt signaling by a pharmacological GSK-3-specific inhibitor. Nat Med. 10, 55-63 
17 Ying, Q. L., Wray, J., Nichols, J., Batlle-Morera, L., Doble, B., Woodgett, J., Cohen, P. and Smith, A. (2008) The ground state of embryonic stem cell self-renewal. Nature. 453, 519-523

18 Houslay, M. D. (2009) Underpinning compartmentalised cAMP signalling through targeted cAMP breakdown. Trends Biochem Sci

19 Michie, A. M., Lobban, M., Muller, T., Harnett, M. M. and Houslay, M. D. (1996)

Rapid regulation of PDE-2 and PDE-4 cyclic AMP phosphodiesterase activity following ligation of the $\mathrm{T}$ cell antigen receptor on thymocytes: analysis using the selective inhibitors erythro-9-(2-hydroxy-3-nonyl)-adenine (EHNA) and rolipram. Cell Signal. 8, 97-110 20 Carson, D. A. and Seegmiller, J. E. (1976) Effect of adenosine deaminase inhibition upon human lymphocyte blastogenesis. J Clin Invest. 57, 274-282

21 Heins, N., Englund, M. C., Sjoblom, C., Dahl, U., Tonning, A., Bergh, C., Lindahl, A., Hanson, C. and Semb, H. (2004) Derivation, characterization, and differentiation of human embryonic stem cells. Stem Cells. 22, 367-376

22 Liu, Y., Song, Z., Zhao, Y., Qin, H., Cai, J., Zhang, H., Yu, T., Jiang, S., Wang, G., Ding, M. and Deng, H. (2006) A novel chemical-defined medium with bFGF and N2B27 supplements supports undifferentiated growth in human embryonic stem cells. Biochem Biophys Res Commun. 346, 131-139

23 Gerrard, L., Rodgers, L. and Cui, W. (2005) Differentiation of human embryonic stem cells to neural lineages in adherent culture by blocking bone morphogenetic protein signaling. Stem Cells. 23, 1234-1241

24 Kane, N. M., Meloni, M., Spencer, H. L., Craig, M. A., Strehl, R., Milligan, G., Houslay, M. D., Mountford, J. C., Emanueli, C. and Baker, A. H. (2010) Derivation of endothelial cells from human embryonic stem cells by directed differentiation: analysis of microRNA and angiogenesis in vitro and in vivo. Arterioscler Thromb Vasc Biol. 30, 13891397

25 Lugnier, C. (2006) Cyclic nucleotide phosphodiesterase (PDE) superfamily: a new target for the development of specific therapeutic agents. Pharmacol Ther. 109, 366-398 26 Boess, F. G., Hendrix, M., van der Staay, F. J., Erb, C., Schreiber, R., van Staveren, W., de Vente, J., Prickaerts, J., Blokland, A. and Koenig, G. (2004) Inhibition of phosphodiesterase 2 increases neuronal cGMP, synaptic plasticity and memory performance. Neuropharmacology. 47, 1081-1092

27 Castro, L. R., Verde, I., Cooper, D. M. and Fischmeister, R. (2006) Cyclic guanosine monophosphate compartmentation in rat cardiac myocytes. Circulation. 113, 2221-2228 28 Antonioli, L., Fornai, M., Colucci, R., Ghisu, N., Da Settimo, F., Natale, G., Kastsiuchenka, O., Duranti, E., Virdis, A., Vassalle, C., La Motta, C., Mugnaini, L., Breschi, M. C., Blandizzi, C. and Del Taca, M. (2007) Inhibition of adenosine deaminase attenuates inflammation in experimental colitis. J Pharmacol Exp Ther. 322, 435-442

29 Hashemi, M, Karami-Tehrani, F., Ghavami, S., Maddika, S. and Los, M. (2005) Adenosine and deoxyadenosine induces apoptosis in oestrogen receptor-positive and negative human breast cancer cells via the intrinsic pathway. Cell Prolif. 38, 269-285

30 Hershfield, M. S. (2005) New insights into adenosine-receptor-mediated immunosuppression and the role of adenosine in causing the immunodeficiency associated with adenosine deaminase deficiency. Eur J Immunol. 35, 25-30

31 Singhal, D. and Anderson, B. D. (1998) Optimization of the local inhibition of intestinal adenosine deaminase (ADA) by erythro-9-(2-hydroxy-3-nonyl)adenine: enhanced oral delivery of an ADA-activated prodrug for anti-HIV therapy. J Pharm Sci. 87, 578-585 32 Tofovic, S. P., Zacharia, L., Carcillo, J. A. and Jackson, E. K. (2001) Inhibition of adenosine deaminase attenuates endotoxin-induced release of cytokines in vivo in rats. Shock. 16, 196-202 
33 Nabhan, C., Gartenhaus, R. B. and Tallman, M. S. (2004) Purine nucleoside analogues and combination therapies in B-cell chronic lymphocytic leukemia: dawn of a new era. Leuk Res. 28, 429-442

34 Cristalli, G., Eleuteri, A., Franchetti, P., Grifantini, M., Vittori, S. and Lupidi, G. (1991) Adenosine deaminase inhibitors: synthesis and structure-activity relationships of imidazole analogues of erythro-9-(2-hydroxy-3-nonyl)adenine. J Med Chem. 34, 1187-1192 35 Da Settimo, F., Primofiore, G., La Motta, C., Taliani, S., Simorini, F., Marini, A. M., Mugnaini, L., Lavecchia, A., Novellino, E., Tuscano, D. and Martini, C. (2005) Novel, highly potent adenosine deaminase inhibitors containing the pyrazolo[3,4-d]pyrimidine ring system. Synthesis, structure-activity relationships, and molecular modeling studies. J Med Chem. 48, $5162-5174$

36 Li, J., Wang, G., Wang, C., Zhao, Y., Zhang, H., Tan, Z., Song, Z., Ding, M. and Deng, H. (2007) MEK/ERK signaling contributes to the maintenance of human embryonic stem cell self-renewal. Differentiation. 75, 299-307

37 Dvorak, P., Dvorakova, D., Koskova, S., Vodinska, M., Najvirtova, M., Krekac, D. and Hampl, A. (2005) Expression and potential role of fibroblast growth factor 2 and its receptors in human embryonic stem cells. Stem Cells. 23, 1200-1211

38 Kinoshita, T., Tada, T. and Nakanishi, I. (2008) Conformational change of adenosine deaminase during ligand-exchange in a crystal. Biochem Biophys Res Commun. 373, 53-57 39 Belles-Isles, M., Chapeau, C., White, D. and Gagnon, C. (1986) Isolation and characterization of dynein ATPase from bull spermatozoa. Biochem J. 240, 863-869 40 Bouchard, P., Penningroth, S. M., Cheung, A., Gagnon, C. and Bardin, C. W. (1981) erythro-9-[3-(2-Hydroxynonyl)] adenine is an inhibitor of sperm motility that blocks dynein ATPase and protein carboxylmethylase activities. Proc Natl Acad Sci U S A. 78, 1033-1036 41 Blackburn, M. R. and Kellems, R. E. (2005) Adenosine deaminase deficiency: metabolic basis of immune deficiency and pulmonary inflammation. Adv Immunol. 86, 1-41 42 Silva, J., Nichols, J., Theunissen, T. W., Guo, G., van Oosten, A. L., Barrandon, O., Wray, J., Yamanaka, S., Chambers, 1. and Smith, A. (2009) Nanog is the gateway to the pluripotent ground state. Cell. 138, 722-737

43 Dravid, G., Ye, Z., Hammond, H., Chen, G., Pyle, A., Donovan, P., Yu, X. and Cheng, L. (2005) Defining the role of Wnt/beta-catenin signaling in the survival, proliferation, and self-renewal of human embryonic stem cells. Stem Cells. 23, 1489-1501

44 Agarwal, R. P., Sagar, S. M. and Parks, R. E., Jr. (1975) Adenosine deaminase from human erythrocytes: purification and effects of adenosine analogs. Biochem Pharmacol. 24, 693-701

45 Agarwal, R. P., Spector, T. and Parks, R. E., Jr. (1977) Tight-binding inhibitors--IV. Inhibition of adenosine deaminases by various inhibitors. Biochem Pharmacol. 26, 359-367 46 Antonini, I., Cristalli, G., Franchetti, P., Grifantini, M., Martelli, S., Lupidi, G. and Riva, F. (1984) Adenosine deaminase inhibitors. Synthesis of deaza analogues of erythro-9(2-hydroxy-3-nonyl)adenine. J Med Chem. 27, 274-278

47 Baker, D. C. and Hawkins, L. D. (1982) Synthesis of inhibitors of adenosinedeaminase - A total synthesis of erythro-3-(adenin-9-yl)-2-nonanol and its isomers from chiral precursors. Journal of Organic Chemistry. 47, 2179-2184

48 Wilson, P. K., Szabados, E., Mulligan, S. P. and Christopherson, R. I. (1998)

Comparative effects of cladribine, fludarabine and pentostatin on nucleotide metabolism in Tand B-cell lines. Int J Biochem Cell Biol. 30, 833-842

\section{FOOTNOTES}


This work was funded by the Stem Cell Technologies Programme Grant from ITI Scotland to MDH, GM, JCM, AHB, DRA.

\section{FIGURE LEGENDS}

Figure 1. hESC morphology and marker expression is maintained and differentiation blocked by EHNA in the absence of FGF during feeder free growth. (a) (i-ii) SA121 hESCs withdrawn from FGF but grown in the presence of EHNA $(10 \mu \mathrm{M})$ remain undifferentiated. (iv-v) Cells grown in the absence of FGF and EHNA show strong differentiated morphology by passage 8 (p8). SA461 hES cells taken from feeder supported culture directly into feeder-free conditions remain undifferentiated in the presence (iii) but not absence (vi) of EHNA. (b) Immunofluoresence of SA121 hES cells shows the absence of SSEA1 (i) and presence of stem cell markers SSEA3 (ii), SSEA4 (iii), TRA1-60 (iv), TRA1-80 (v) and POU5F1 (vi). Specific antibodies are in green and DAPI in blue. Scale bars, $100 \mu \mathrm{m}$. (c) qRT-PCR was used to analyse the samples at p8. Gene expression is relative to expression in normal feeder free cells grown with FGF. Student $t$ tests compared data between experimental groups. Asterisks indicate that cells treated with EHNA are statistically significant in comparison to cells treated with neither EHNA or FGF. ${ }^{*}, P<0.05 ;{ }^{* *}, P<0.01 ; * * *, P<0.001$

Figure 2. The ability of EHNA to inhibit differentiation is reversible. After growth for 12 passages in the presence of EHNA $(10 \mu \mathrm{M})$ instead of FGF SA121 hESCs were transferred to gelatin and differentiated for 4 weeks in 20\% fetal calf serum: (a) immunostaining for AFP (green) and DAPI (blue), (b) immunostaining for PAX6 (red) and DAPI (blue), (c) immunostaining for smooth muscle actin (green) and DAPI (blue). Scale bars, $100 \mu \mathrm{m}$.

Figure 3. Prior growth in EHNA does not block subsequent differentiation. Neuronal differentiations were initiated from SA121 hESCs previously grown with either FGF or EHNA (10 $\mu \mathrm{M}$ ) for 5 passages: (a) NANOG expression during neuronal differentiation, (b) PAX6 expression during neuronal differentiation, (c) immunostaining of neuronal differentiation (day 16) after prior growth in EHNA, (d) immunostaining of cells neuronal differentiation (day 16) after prior growth in FGF. All images are representative fields stained for POU5F1 (green), Pax6 (red) and DAPI (blue). Scale bars, $100 \mu \mathrm{m}$.

Figure 4. Directed differentiation is inhibited by the presence of EHNA. (a) SA121 cells were induced to differentiate towards neural lineages with increasing concentrations of EHNA $(0-10 \mu \mathrm{M})$. Immunostaining is of POU5F1 (green), PAX6 (red) and DAPI (blue). Scale bars, 100 $\mu \mathrm{m}$. qRT-PCR analysis of (b) Nanog, (c) POUF51, (d) ZFP42, and (e) PAX6 expression was analysed throughout a neural differentiation with and without EHNA $(10 \mu \mathrm{M})$ present continually. (f) Expression of NANOG and PAX6 was analysed by qRT-PCR at 4 weeks and 6 weeks post neural differentiation induction. EHNA was either present throughout (+EHNA; $10 \mu \mathrm{M})$ or absent (-EHNA) from the differentiations $(\mathrm{ND}=$ neural differentiation).

Figure 5. EHNA does not maintain transcription of hESC markers through inhibition of PDE2, increased phosphorylated metabolites of adenosine or adenosine receptor activation. SA121 hESCs underwent passive differentiation for 14 days (with and without compound addition) and were subsequently analysed by qRT-PCR. (a) and (b) Treatment with EHNA and PDE inhibitors. (c) Treatment with adenosine kinase inhibitor, AMPK agonist and adenosine receptor agonist and antagonist. (d) Treatment with EHNA and inhibitors of each specific adenosine receptor. Data represents mean \pm SEM from three experiments. For (a) and (b) the value 1 was set to level of expression in undifferentiated hESCS whereas in (c) and (d) the value 1 was set to the expression level in the untreated control samples. All other values were calculated respectively. EHNA - erythro-9-(2hydroxy-3-nonyl)adenine (10 $\mu \mathrm{M})$; IBMX - 3-isobutyl-1-methylxanthine $(100 \mu \mathrm{M})$; BAY - BAY-60$7550(5 \mu \mathrm{M})$; AKI - adenosine kinase inhibitor (ABT-702) $(5 \mu \mathrm{M})$, AICAR - 5-aminoimidazole-4carboxamide-1- $\beta$-D-ribofuranoside $(0.5 \mathrm{mM})$; NECA - adenosine-5'- $N$-ethylcarboxamide $(10 \mu \mathrm{M})$, THEO - theophylline $(10 \mu \mathrm{M})$; CPX - 8-cyclopentyl-1,3-dipropylxanthine $(10 \mu \mathrm{M}), \mathrm{SCH}-\mathrm{SCH}$ 
$58261(10 \mu \mathrm{M}), \mathrm{M} 3$ - MRS3777 $(10 \mu \mathrm{M}), \mathrm{M} 1$ - MRS1754 $(10 \mu \mathrm{M})$. Student $\mathrm{t}$ tests compared data between experimental groups. Asterisks indicate that cells treated with EHNA are statistically significant in comparison to the untreated control group. *, $P<0.05 ;{ }^{* *}, P<0.01 ; * * *, P<0.001$.

Figure 6. The effect of known ADA inhibitors and related compounds on the expression of NANOG and PAX6 in SA121 hESCs under differentiation conditions. Compounds which maintained, after 14 days of differentiation, at least $50 \%$ of the level of NANOG-expression in comparison to EHNA and those that inhibited the expression of PAX6 to 50\% or less than the value of the untreated controls were considered to have an EHNA-like effect. Those which had a single effect, to these levels, on either PAX6 or NANOG were considered to have a partial-EHNA effect. All compounds were tested at $10 \mu \mathrm{M}$. All compound structures are defined in the Supplementary Figure 4. [44-48]Figure 7. The differentiation blocking effect of EHNA is not associated with adenosine deaminase inhibition. SA121 hESCs underwent passive differentiation for 14 days (with and without compound addition) and subsequently analysed by qRT-PCR. Data represents mean $\neq \mathrm{SEM}$ from three experiments. Expression is relative to expression in the untreated control samples. Con - untreated; EHNA - erythro-9-(2-hydroxy-3-nonyl)adenine; HWC-57 - deoxy-EHNA; HWC-30 - 2-nonyl-2Hpyrazolo[3,4- $d$ ]pyrimidin-4-amine hydrochloride; HWC-31 - 1-nonyl-1H-pyrazolo[3,4- $d$ ]pyrimidin-4amine hydrochloride; HWC-33 - 1-undecyl-1H-pyrazolo[3,4- $d]$ pyrimidin-4-amine hydrochloride; Pento - pentostatin. All compounds were used at $10 \mu \mathrm{M}$. Student $t$ tests compared data between experimental groups. Asterisks indicate that cells treated with EHNA are statistically significant in comparison to the untreated control group. ${ }^{*}, P<0.05$; **, $P<0.01$, ***, $P<0.001$.

Figure 8. Standard hESC morphology and expression profile is maintained by HWC-57 and HWC-64 in the absence of FGF for up to 10 passages. (a) Phase images of SA121 hESCs grown for 10 passages in full feeder free media containing FGF (FF), or media containing no FGF (NF), or media containing no FGF but supplemented with either $10 \mu \mathrm{M}$ HWC-57 or HWC-64. (b) Analysis of gene expression from cells passaged in the conditions referred to in (a). Gene expression is relative to GAPDH. Scale bars, $100 \mu \mathrm{m}$. 
Figure 1.

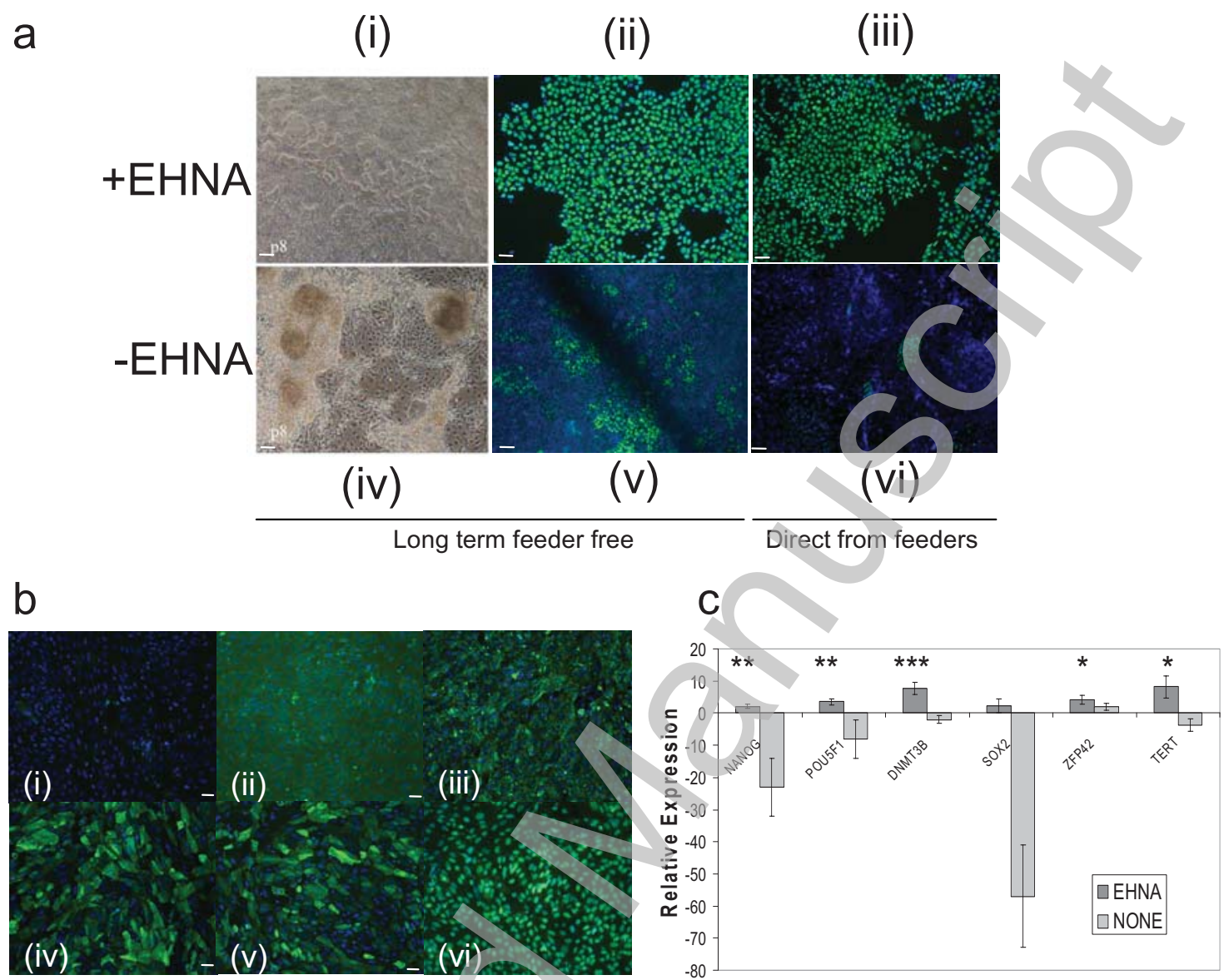


Figure 2.

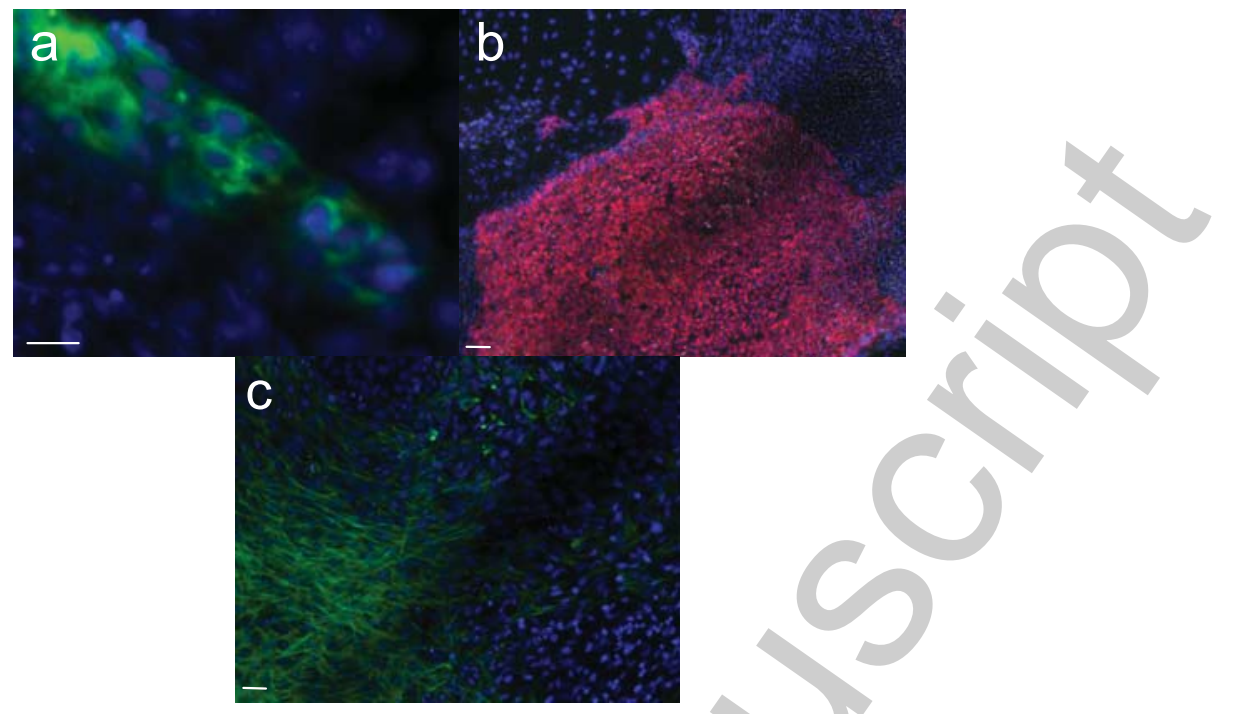


Figure 3.
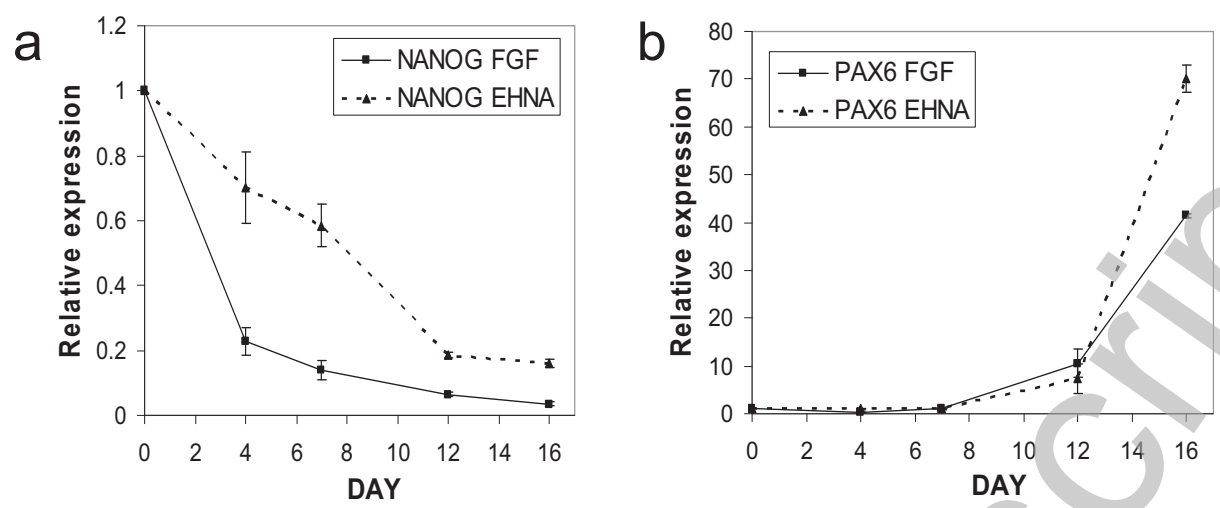

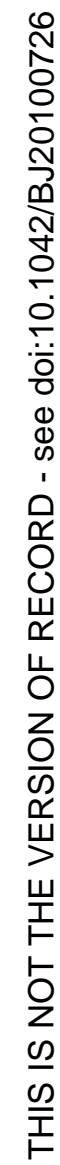

\section{C}

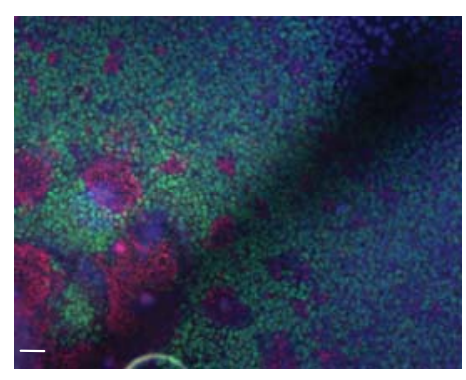

d

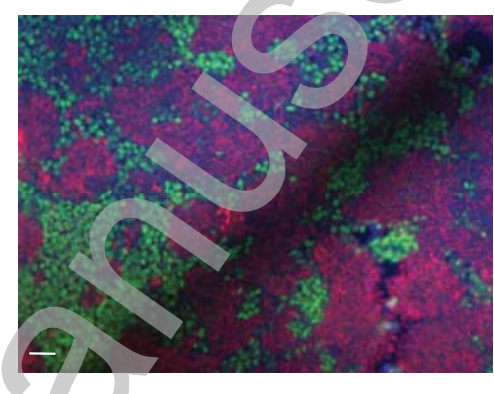


Figure 4 .

a

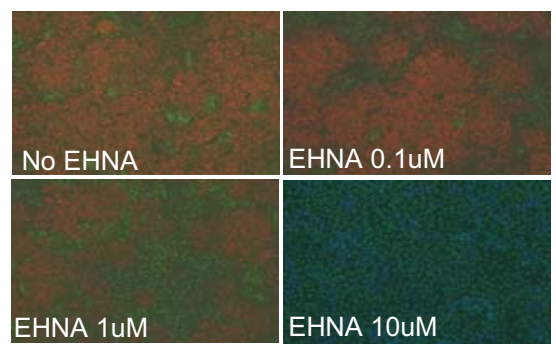

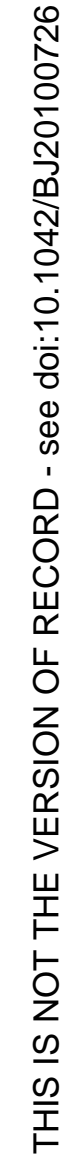
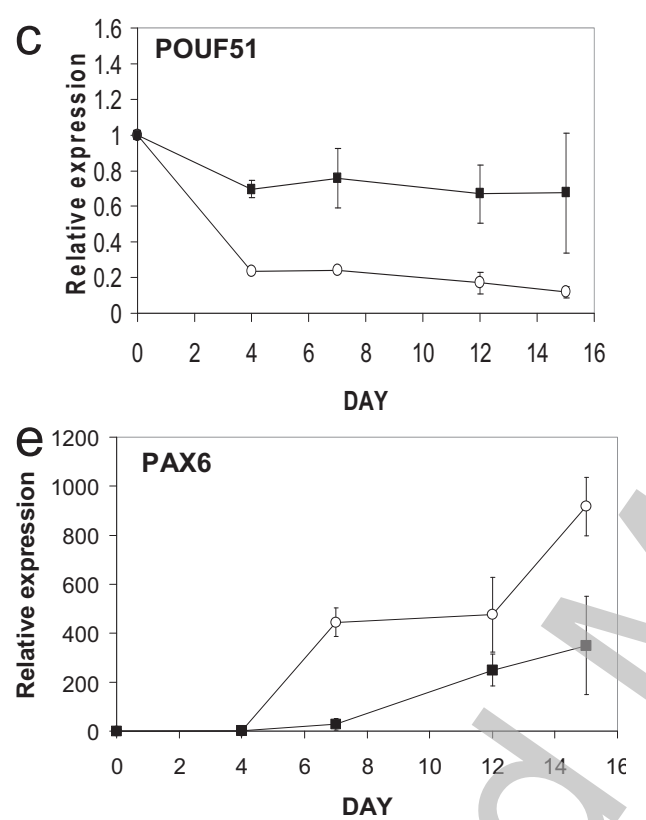

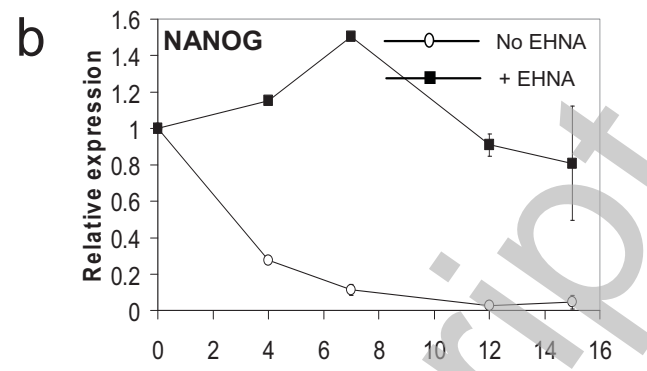

DAY

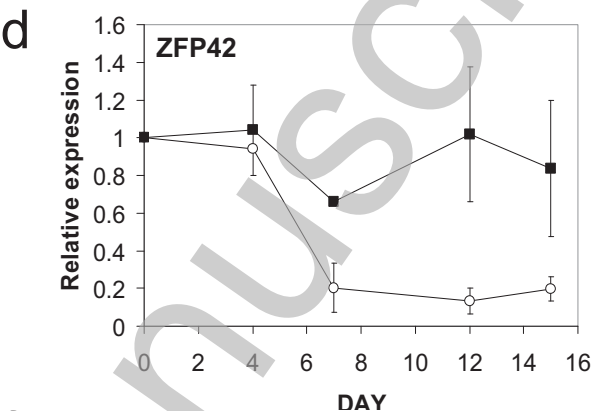

f

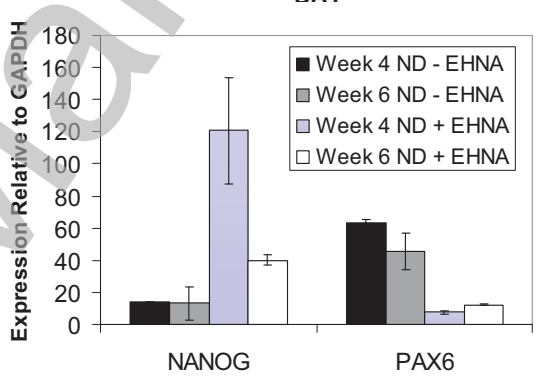


Figure 5.
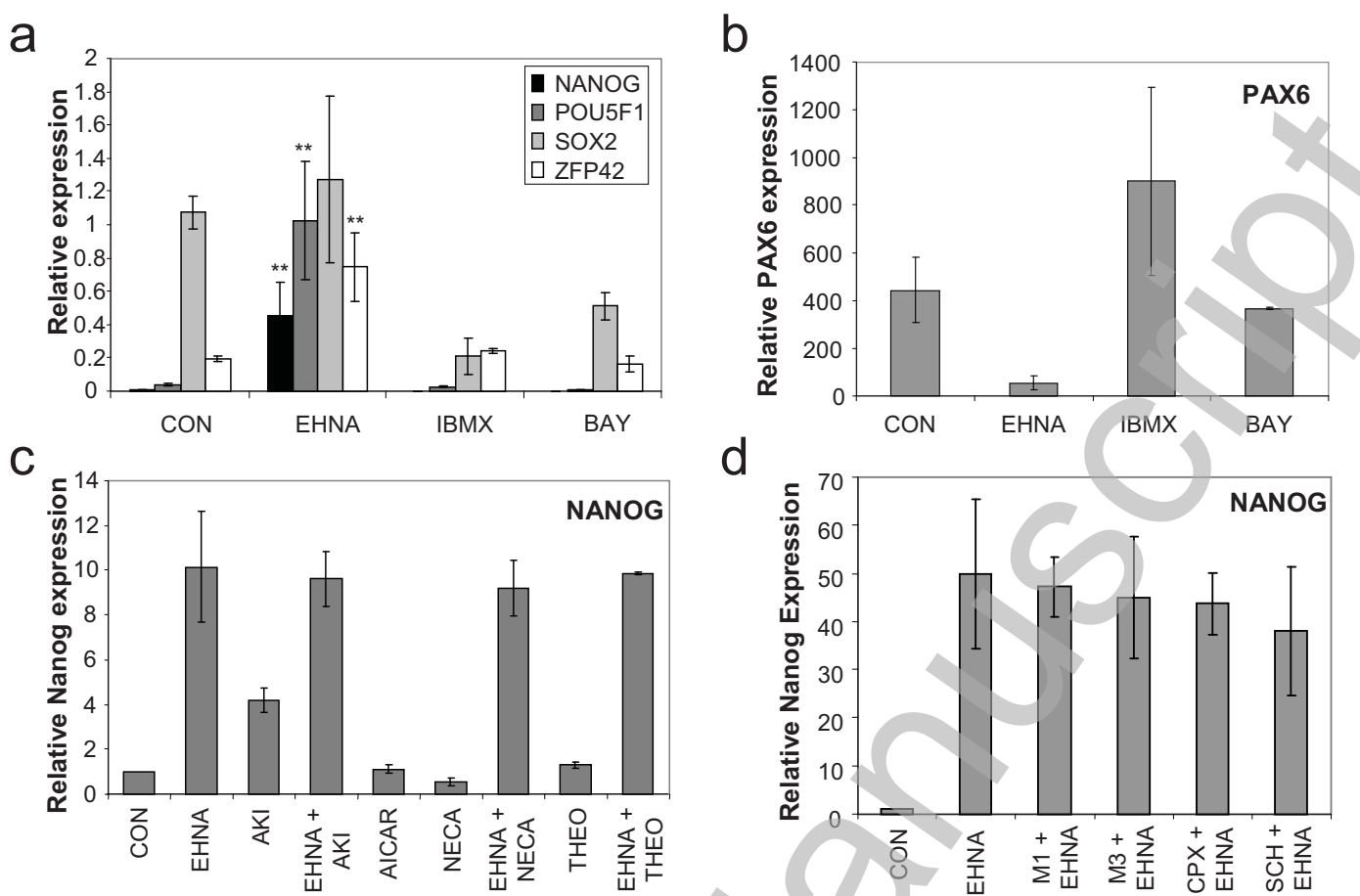

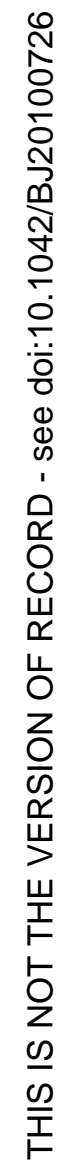

Licenced copy. Copying is not permitted, except with prior permission and as allowed by law. (C) 2010 The Authors Journal compilation (c) 2010 Portland Press Limited 
Figure 6.

a

\begin{tabular}{|c|c|c|c|c|c|}
\hline & $\begin{array}{l}\text { Full } \\
\text { EHNA } \\
\text { effect }\end{array}$ & $\begin{array}{c}\text { PARTIAL } \\
\text { EHNA } \\
\text { EFFECT } \\
\text { (NANOG) }\end{array}$ & $\begin{array}{c}\text { PARTIAL } \\
\text { EHNA } \\
\text { EFFECT } \\
\text { (PAX6) }\end{array}$ & $\begin{array}{c}\text { NO } \\
\text { EHNA } \\
\text { EFFECT }\end{array}$ & $\begin{array}{c}\text { Reported } \\
\text { adenosine } \\
\text { deaminase } \\
\text { inhibition }\end{array}$ \\
\hline EHNA & $\mathrm{x}$ & & & & $K_{\mathrm{i}} 7 \mathrm{nM}^{34}$ \\
\hline HWC-06 & $\mathrm{x}$ & & & & $K_{\mathrm{i}} 0.13 \mathrm{nM}^{35}$ \\
\hline HWC-25 & $x$ & & & & ND \\
\hline HWC-30 & $x$ & & & & Inactive ${ }^{35}$ \\
\hline HWC-31 & $\mathrm{x}$ & & & & $K_{\mathrm{i}} 8.1 \mathrm{nM}^{35}$ \\
\hline HWC-33 & $x$ & & & & $K_{\mathrm{i}} 0.47 \mathrm{nM}^{35}$ \\
\hline HWC-40 & $x$ & & & & ND \\
\hline HWC-41 & $x$ & & & & $K_{\mathrm{i}} 530 \mathrm{nM}^{35}$ \\
\hline HWC-46 & $\mathrm{x}$ & & & & $K_{\mathrm{i}} 0.76 \mathrm{nM}^{47}$ \\
\hline HWC-57 & $\mathrm{x}$ & & & & ND \\
\hline HWC-62 & $x$ & & & & ND \\
\hline HWC-64 & $x$ & & & & ND \\
\hline HWC-08 & & & $\mathrm{x}$ & & $K_{\mathrm{i}} 1.2 \mathrm{uM}^{46}$ \\
\hline HWC-09 & & & $x$ & & $K_{\mathrm{i}} 10 \mathrm{nM}^{46}$ \\
\hline HWC-10 & & & $x$ & & ND \\
\hline HWC-12 & & & $x$ & & $K_{\mathrm{i}} 840 \mathrm{nM}^{46}$ \\
\hline HWC-13 & & & $x$ & & ND \\
\hline HWC-16 & & & $\mathrm{x}$ & & ND \\
\hline HWC-17 & & & $x$ & & ND \\
\hline HWC-21 & & & $x$ & & $K_{\mathrm{i}} 73 \mathrm{uM}^{48}$ \\
\hline HWC-24 & & & $\mathrm{x}$ & & ND \\
\hline HWC-26 & & & $x$ & & ND \\
\hline HWC-27 & & & $x$ & & ND \\
\hline HWC-34 & & & $x$ & & ND \\
\hline HWC-35 & & & $x$ & & ND \\
\hline HWC-52 & & & $x$ & 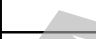 & $K_{\mathrm{i}} 62 \mathrm{nM}^{47}$ \\
\hline HWC-05 & & & & $x$ & $K_{\mathrm{i}} 550 \mathrm{nM}^{46}$ \\
\hline HWC-07 & & & & $x$ & $K_{i} 368 n^{34}$ \\
\hline HWC-14 & & & & $\mathrm{x}$ & ND \\
\hline HWC-15 & & & & $x$ & ND \\
\hline HWC-18 & & & & $x$ & $K_{\mathrm{i}} 17 \mathrm{uM}^{44}$ \\
\hline HWC-36 & & & & $x$ & $K_{\mathrm{i}} 35 \mathrm{nM}^{34}$ \\
\hline HWC-44 & & & & $x$ & ND \\
\hline HWC-60 & & & & $x$ & ND \\
\hline pentostatin & & & & $x$ & $\begin{array}{c}K_{\mathrm{i}} 2.5 \mathrm{pM}^{45} \\
K_{\mathrm{i}} 22 \mathrm{pM}^{48}\end{array}$ \\
\hline
\end{tabular}

b

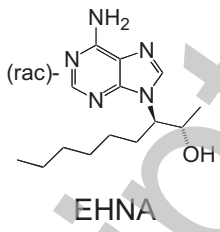

HWC-57

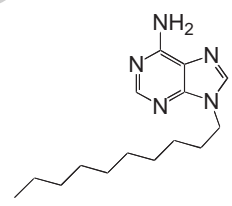

HWC-64

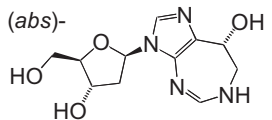

pentostatin (deoxycoformycin) 
Figure 7.
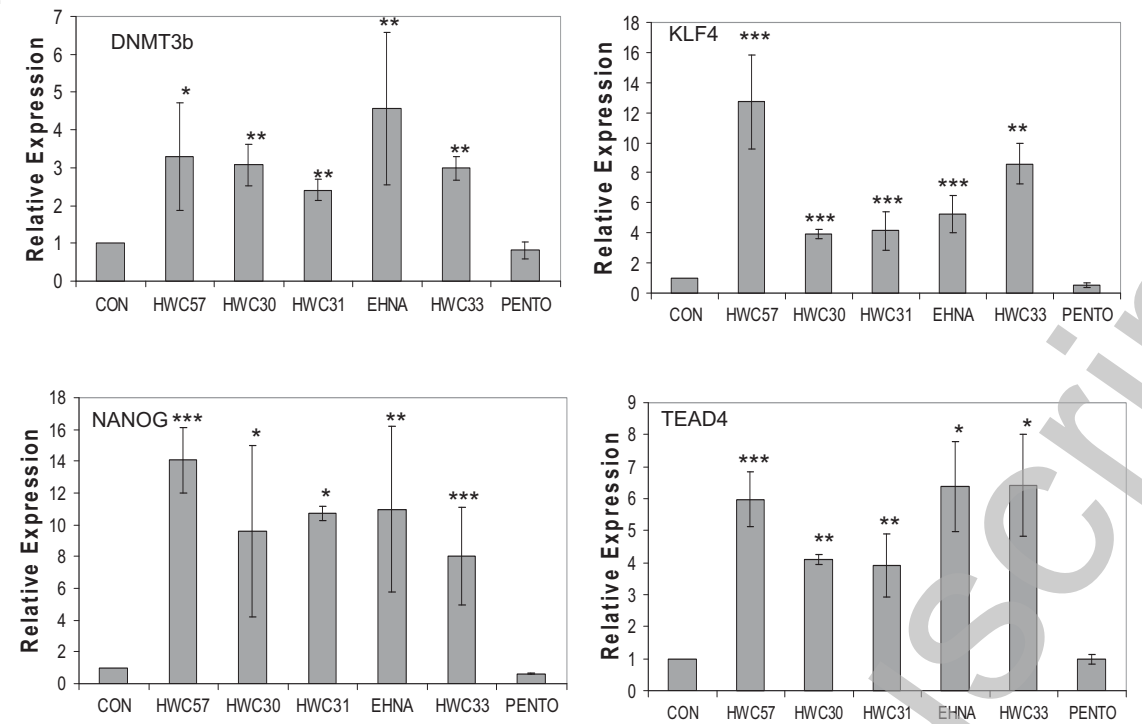

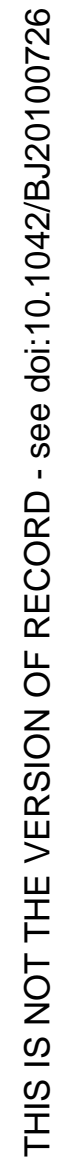
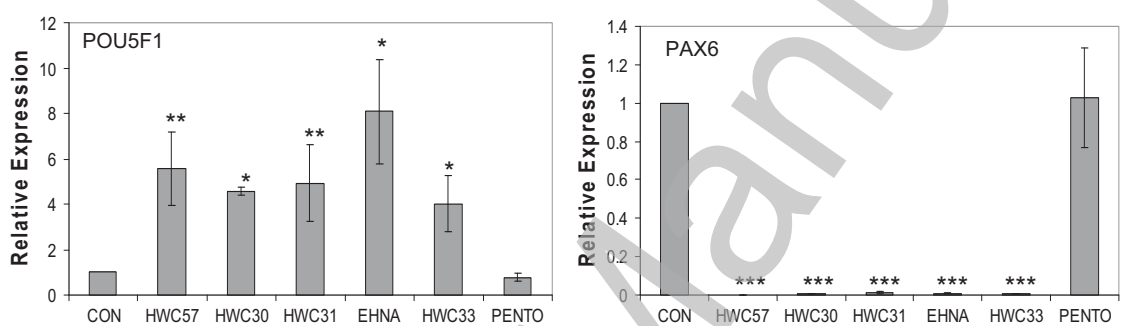
Figure 8.

a

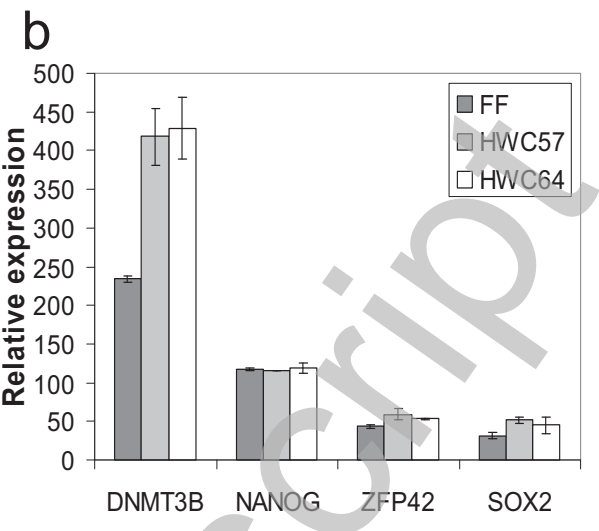

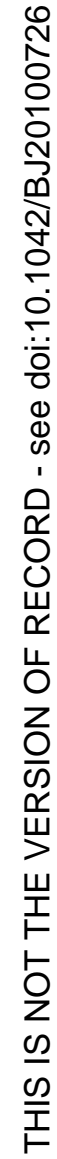
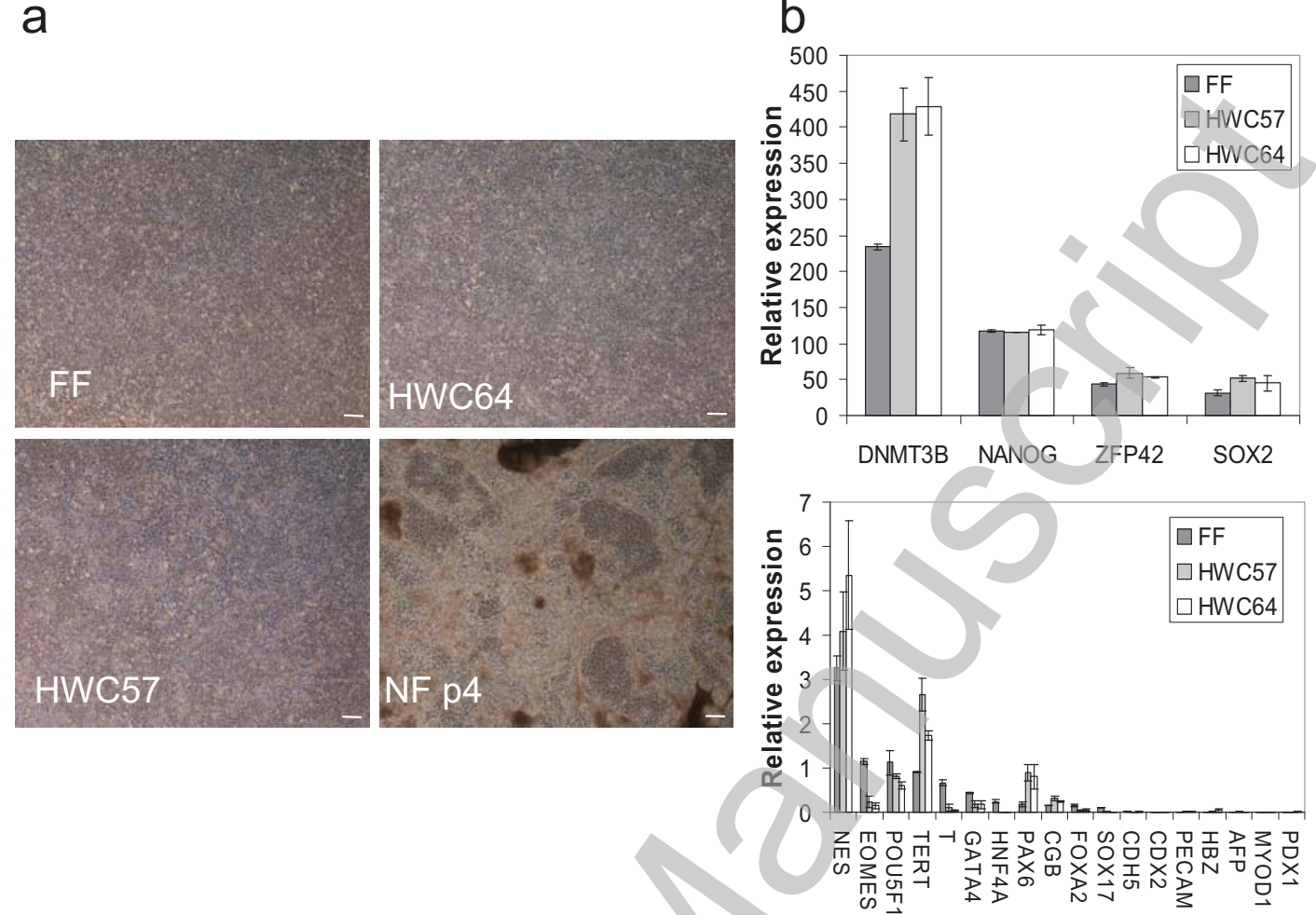